\author{
SUPPORTING INFORMATION
}

\title{
ATRP of tert-Butoxycarbonylaminomethyl Acrylate (tBAMA): Well-Defined Precursors for Polyelectrolytes of Tunable Charge
}

Mark Billing, Felix H. Schacher*

Laboratory of Organic and Macromolecular Chemistry, Friedrich-Schiller-University Jena, Humboldtstraße 10, D-07743 Jena, Germany

felix.schacher@uni-jena.de

Jena Center for Soft Matter (JCSM), Friedrich-Schiller-University Jena, Philosophenweg 7, D-07743 Jena, Germany 
Kinetic study dNbpy/Cu(I):

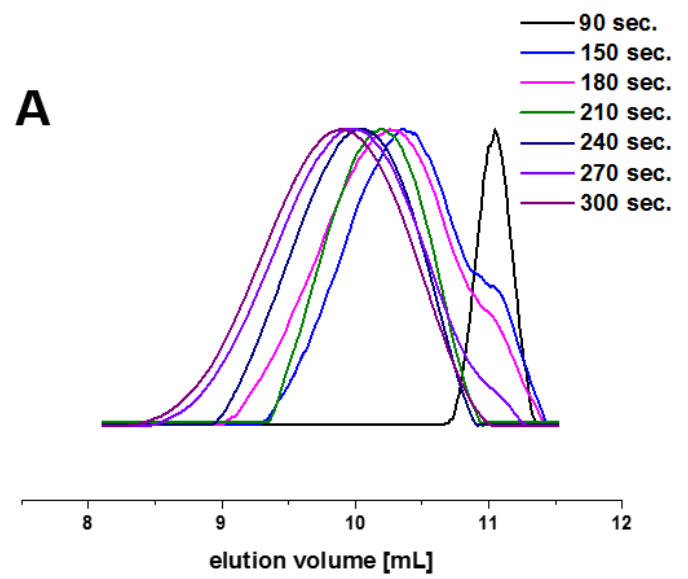

B

\begin{tabular}{cccc}
\hline Time $[$ sec. $]$ & $\mathbf{M}_{n}$ & $\mathbf{M}_{w}$ & Ð \\
0 & - & - & - \\
90 & 800 & 860 & 1.19 \\
150 & 1550 & 1890 & 1.21 \\
180 & 1800 & 2200 & 1.24 \\
210 & 2100 & 2300 & 1.10 \\
240 & 2400 & 2800 & 1.15 \\
270 & 2400 & 3000 & 1.25 \\
300 & 2700 & 3300 & 1.22 \\
\hline
\end{tabular}

Figure S1: ATRP of $t$ BAMA with dNbpy in anisole $[75 \% \mathrm{v} / \mathrm{v}]$ at $50{ }^{\circ} \mathrm{C}$ : A) SEC elution traces, B) corresponding SEC data; $\mathrm{SEC}$ [( $\mathrm{CHCl}_{3} / i$-PrOH / TEA. (94/2/4): PS calibration].

Kinetic study $\mathrm{Me}_{6} \mathrm{TREN} / \mathrm{Cu}(\mathrm{I})$ :

A

B

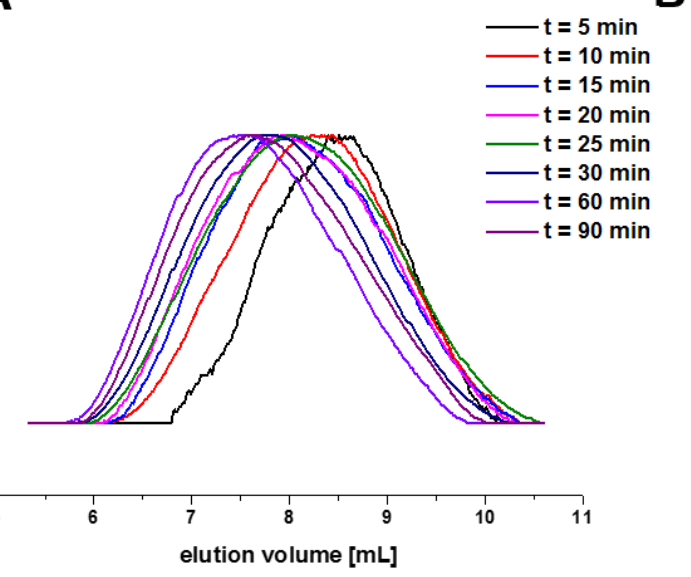

\begin{tabular}{cccc}
\hline Time [min] & $\mathbf{M}_{n}$ & $\mathbf{M}_{w}$ & Ð \\
0 & - & - & - \\
5 & $\mathbf{8 9 0 0}$ & 12600 & 1.41 \\
10 & 10000 & 16600 & 1.65 \\
15 & 11000 & 19100 & 1.73 \\
20 & 11400 & 20400 & 1.78 \\
25 & 10600 & 19900 & 1.89 \\
30 & 13300 & 23900 & 1.79 \\
60 & 17300 & 30500 & 1.76 \\
90 & 14800 & 26700 & 1.80 \\
\hline
\end{tabular}

Figure S2: ATRP of $t$ BAMA with Me ${ }_{6}$ TREN in anisole $\left[\begin{array}{llllll}4 & \% & v / v\end{array}\right]$ at $30 \quad{ }^{\circ} \mathrm{C}$ ([tBAMA $\left.] /[\mathrm{Cu}(\mathrm{I})] /\left[\mathrm{Me}_{6} \mathrm{TREN}\right] /[\mathrm{MeBiB}]=100 / 1 / 1 / 1\right):$ A) SEC elution traces, B) corresponding SEC data, SEC $\left[\left(\mathrm{CHCl}_{3} / i\right.\right.$-PrOH / TEA. (94/2/4): PS calibration]. 
Kinetic study Me $\mathrm{M}_{6} \mathrm{TREN} / \mathrm{Cu}(\mathrm{I})$ :

A

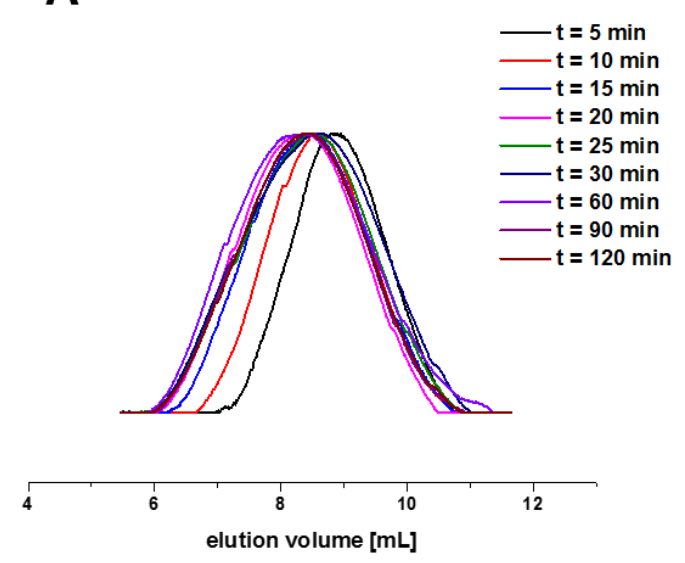

B

\begin{tabular}{cccc}
\hline Time [min] & $\mathbf{M}_{n}$ & $\mathbf{M}_{w}$ & Ð \\
0 & - & - & - \\
5 & $\mathbf{5 8 0 0}$ & $\mathbf{8 4 0 0}$ & $\mathbf{1 . 4 5}$ \\
10 & 7200 & 11500 & $\mathbf{1 . 5 9}$ \\
15 & $\mathbf{8 0 0 0}$ & $\mathbf{1 4 7 0 0}$ & $\mathbf{1 . 8 3}$ \\
20 & 9200 & 17200 & $\mathbf{1 . 8 6}$ \\
25 & 7900 & 16500 & 2.09 \\
30 & 7100 & 15900 & 2.23 \\
60 & 7600 & 17800 & 2.33 \\
90 & $\mathbf{8 4 0 0}$ & 16800 & 1.98 \\
120 & $\mathbf{8 3 0 0}$ & 16700 & $\mathbf{2 . 0 8}$ \\
\hline
\end{tabular}

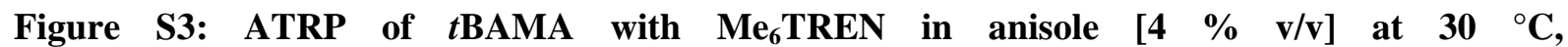
$\left([\right.$ BAMA $\left.] /[\mathrm{Cu}(\mathrm{I})] /\left[\mathrm{Me}_{6} \mathrm{TREN}\right] /[\mathrm{MeBiB}]=50 / 1 / 1 / 1\right)$ : A) SEC elution traces; $\left.\mathrm{B}\right)$ corresponding SEC data, SEC $\left[\left(\mathrm{CHCl}_{3} / i\right.\right.$-PrOH / TEA. (94/2/4): PS calibration]. 


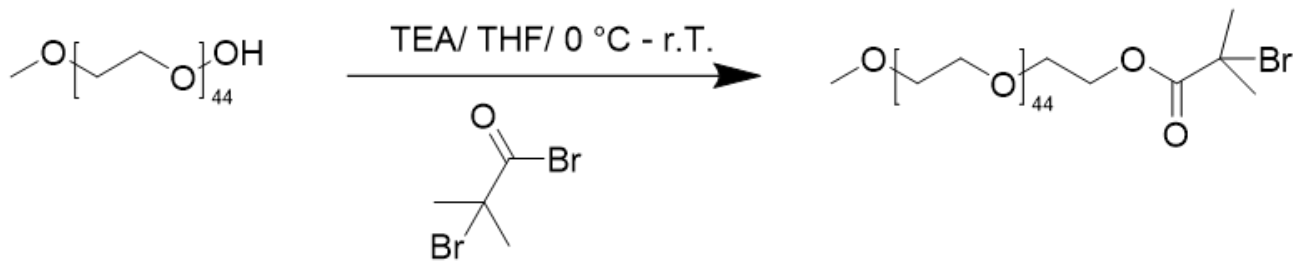

Scheme S1: Endgroup functionalization of $\mathrm{PEO}_{44}-\mathrm{OH}$ to $\mathrm{PEO}_{44}-\mathrm{Br}$.

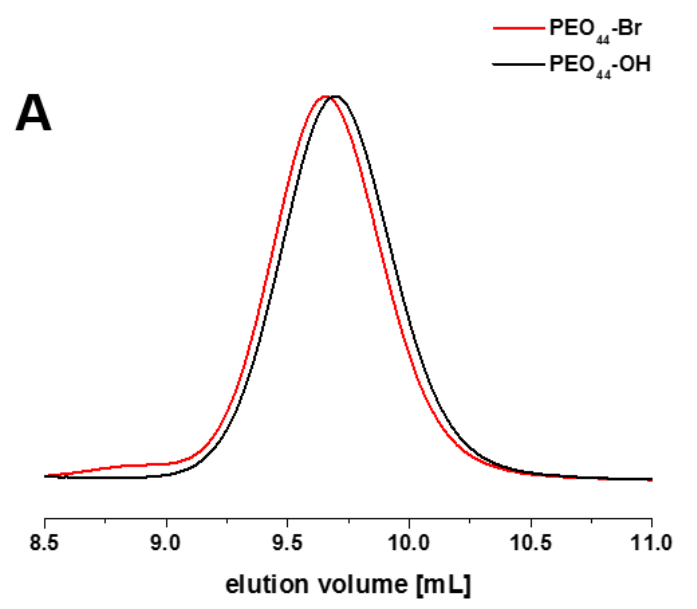

B

\begin{tabular}{ccc}
\hline Polymer & $\mathrm{M}_{n}\left[\mathrm{~g} \mathrm{~mol}^{-1}\right]$ & Đ \\
PEO $_{44}-\mathrm{OH}$ & 1800 & 1.04 \\
PEO $_{44}-\mathrm{Br}$ & 1900 & 1.04 \\
\hline
\end{tabular}

Figure S4: Endgroup functionalization of $\mathrm{PEO}_{44}-\mathrm{OH}$ to $\mathrm{PEO}_{44}-\mathrm{Br}$ : A) SEC elution traces; B) corresponding SEC data, SEC [(CHCl $/$ i-PrOH / TEA. (94/2/4): PS calibration]. 
Synthesis of Macroinitiators:

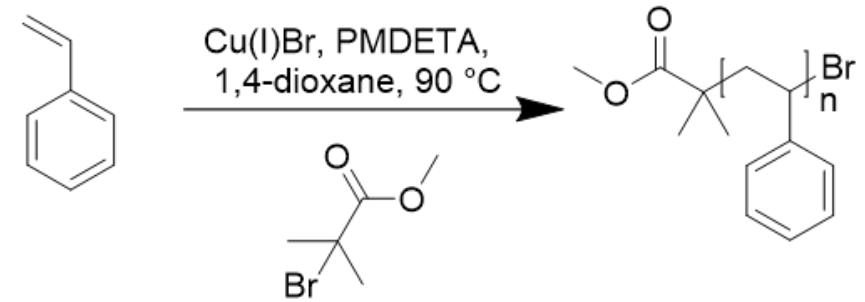

Scheme S2: Synthesis of $\mathbf{P S}_{30}$-Br macroinitiator by ATRP.

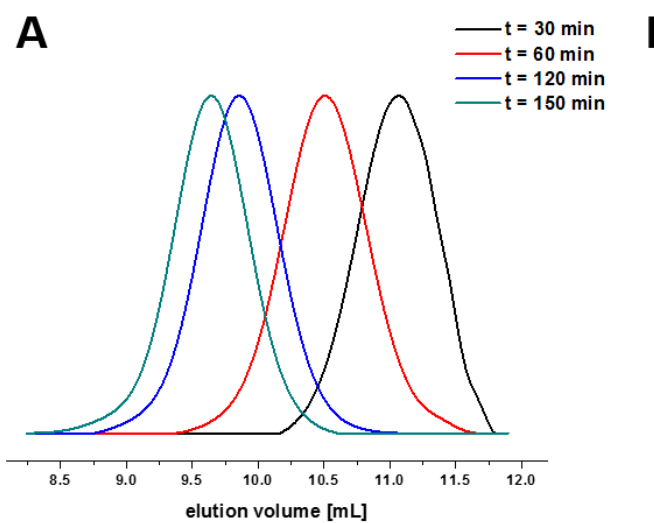

B

\begin{tabular}{cccc}
\hline Time $[\mathrm{min}]$ & $\mathrm{M}_{n}$ & $\mathrm{M}_{w}$ & $\mathrm{D}$ \\
0 & - & - & - \\
30 & 700 & 800 & 1.16 \\
60 & 1200 & 1400 & 1.12 \\
120 & 2400 & 2600 & 1.08 \\
150 & 3000 & 3100 & 1.08 \\
\hline
\end{tabular}

Figure S5: ATRP of styrene with PMDETA in anisole at $90^{\circ} \mathrm{C}$ : A) SEC elution traces; B) corresponding SEC data, SEC [(CHCl $/$ / i-PrOH / TEA. (94/2/4): PS calibration]. 


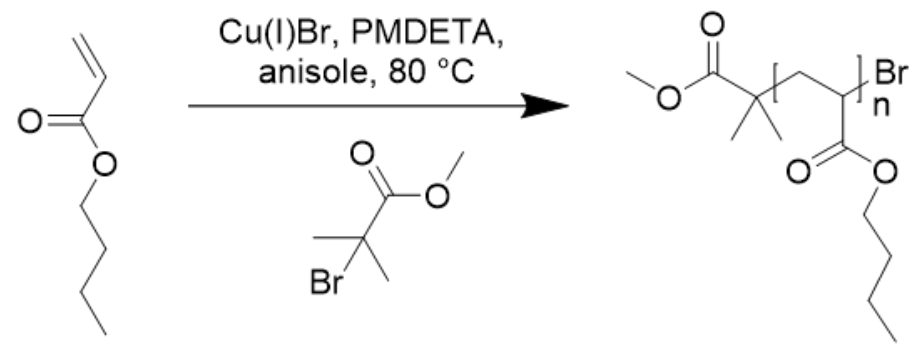

Scheme S3: Synthesis of PnBA-Br macroinitiator by ATRP.

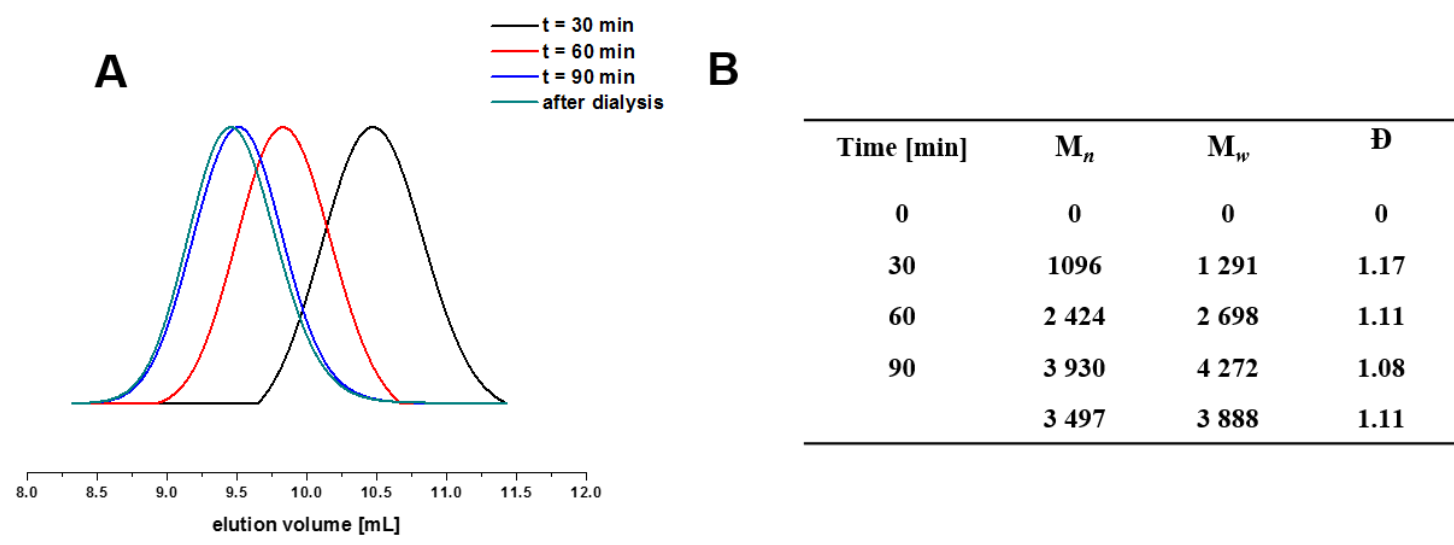

Figure S6: ATRP of $n \mathrm{BA}$ with PMDETA in anisole at $80^{\circ} \mathrm{C}$ : A) SEC elution traces; B) corresponding SEC data, SEC [(CHCl 3 / i-PrOH / TEA. (94/2/4): PS calibration]. 


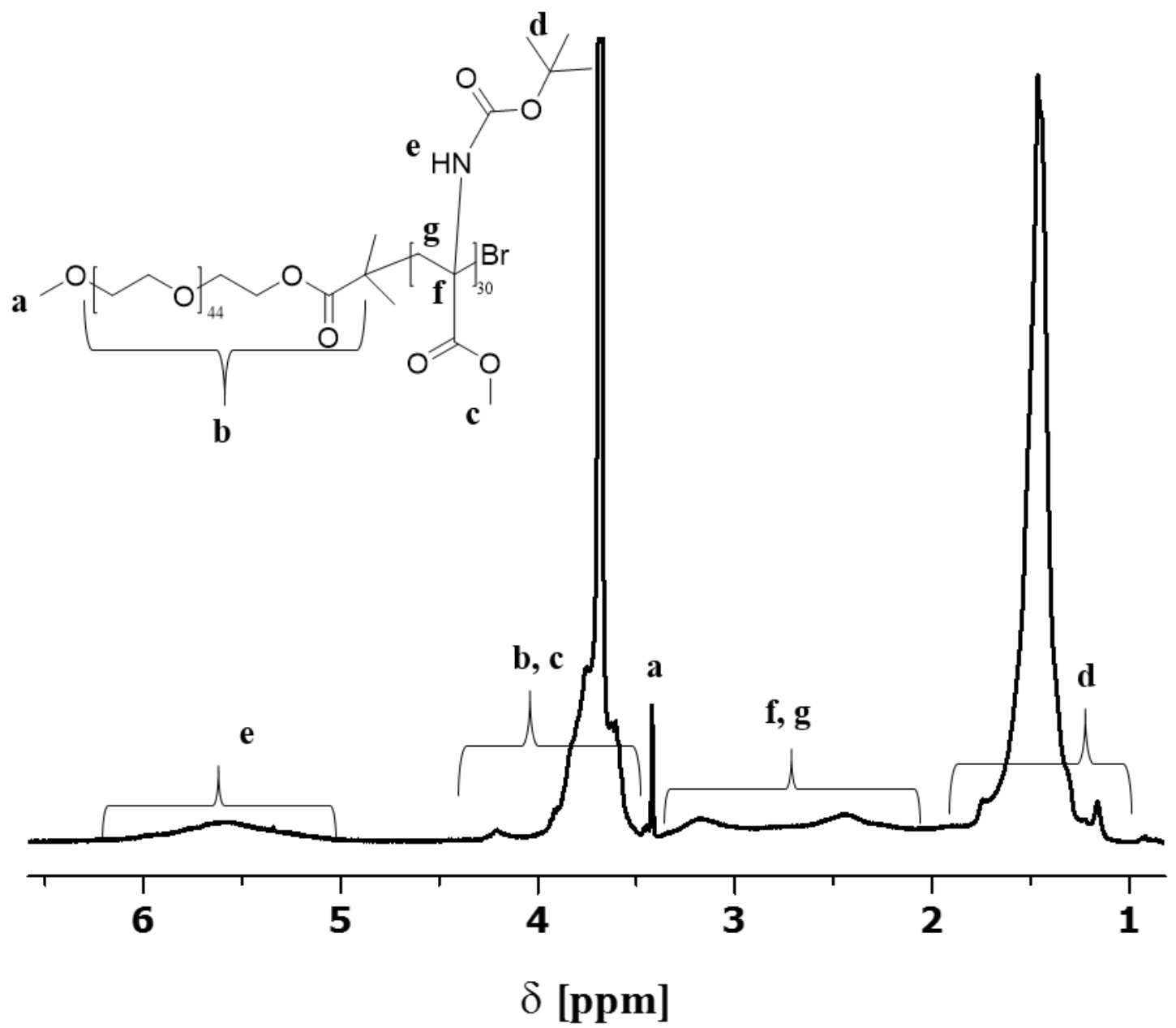

Figure S7: ${ }^{1} \mathrm{H}-\mathrm{NMR}$ of $\mathrm{PEO}_{44}-b$-PtBAMA ${ }_{30}$ in $\mathrm{CDCl}_{3} 300 \mathrm{MHz}$. 


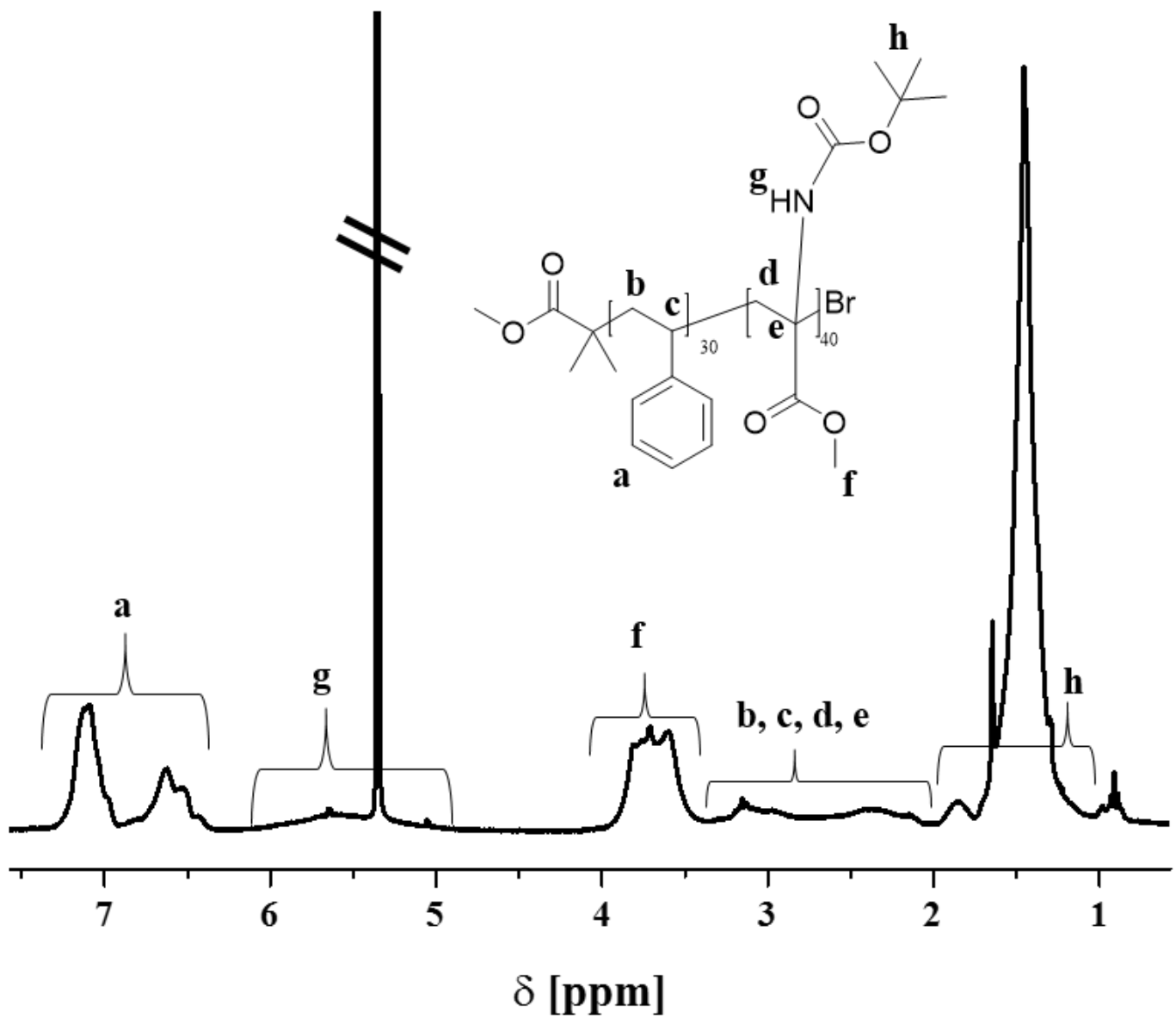

Figure S8: ${ }^{1} \mathrm{H}-\mathrm{NMR}$ of $\mathrm{PS}_{30}-b$-PtBAMA ${ }_{40}$ in $\mathrm{CD}_{2} \mathrm{Cl}_{2} 300 \mathrm{MHz}$. 


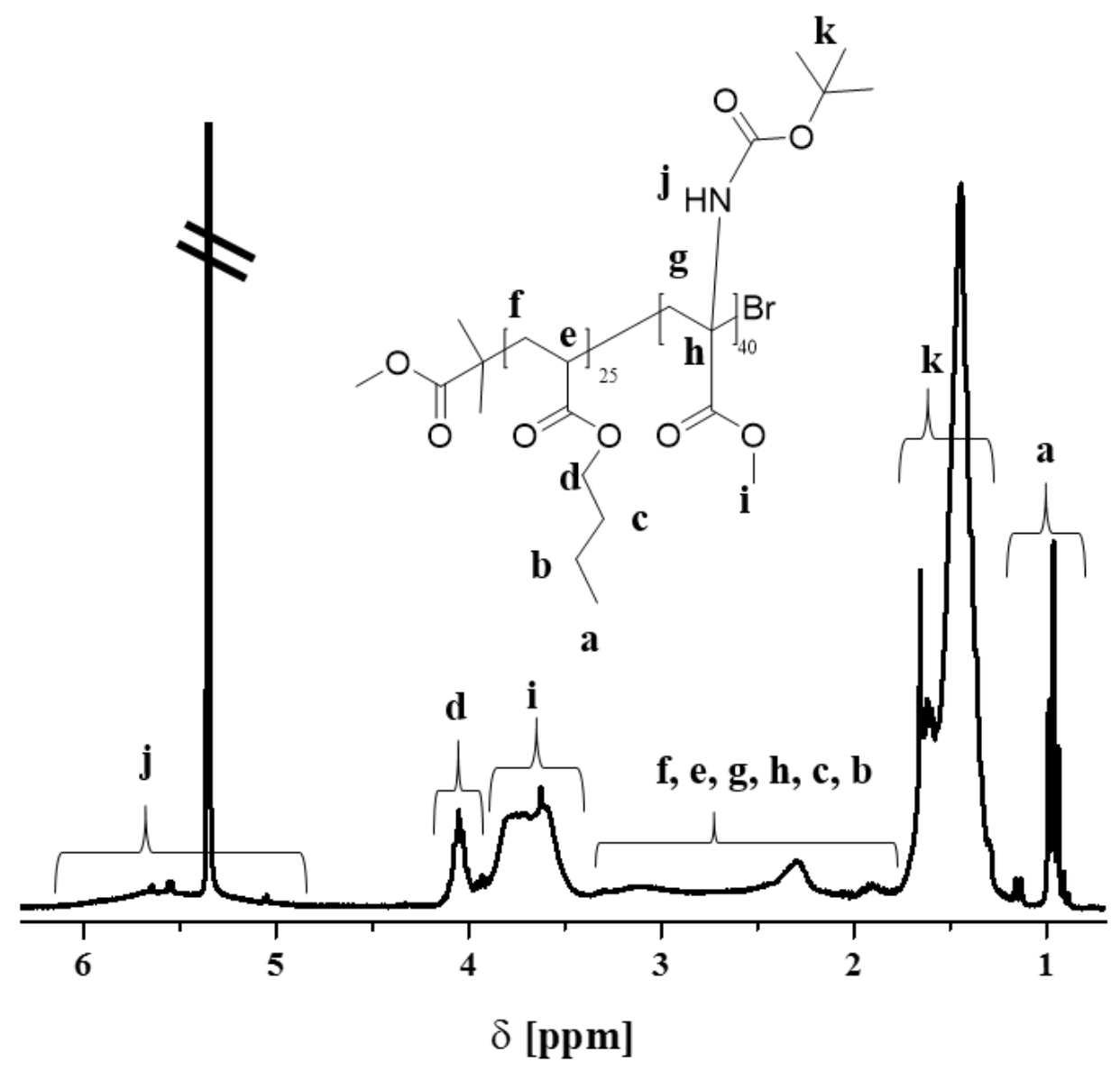

Figure S9: ${ }^{1} \mathrm{H}-\mathrm{NMR}$ of $\mathrm{PnBA}_{25}-b$-PtBAMA $A_{50}$ in $\mathrm{CD}_{2} \mathrm{Cl}_{2} 300 \mathrm{MHz}$. 


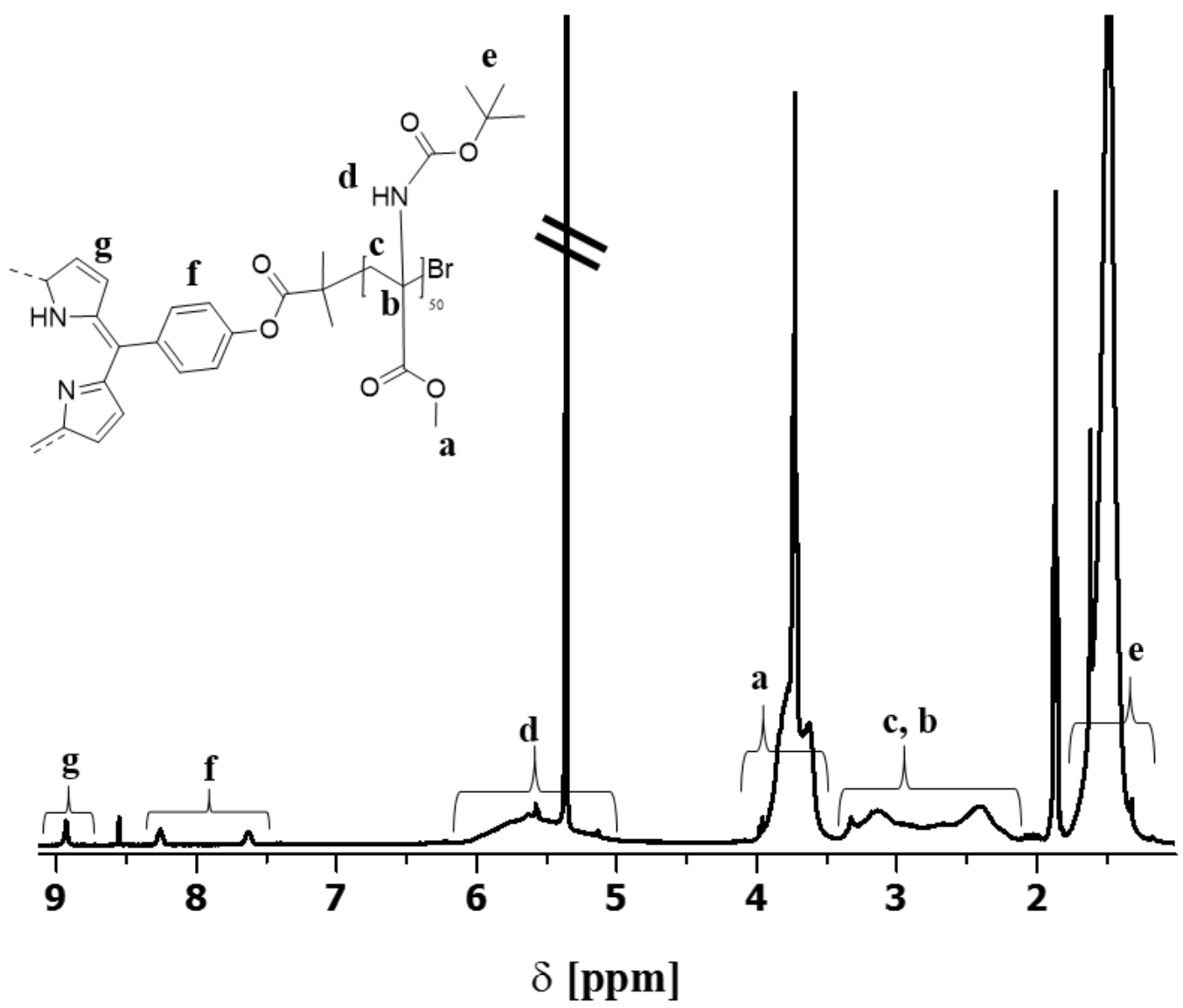

Figure S10: ${ }^{1} \mathrm{H}-\mathrm{NMR}$ of [TTP-P $\left.t \mathrm{BAMA}_{50}\right]_{4}$ in $\mathrm{CD}_{2} \mathrm{Cl}_{2} 300 \mathrm{MHz}$. 
Table S1: $\mathbf{M}_{n \text {, Theo }}, M_{n}$, and yields of selected polymers and block copolymers.

\begin{tabular}{|c|c|c|c|c|c|c|}
\hline Entry & Polymer & $\mathrm{M}_{n}\left[\mathrm{~g} \mathrm{~mol}^{-1}\right]^{\mathrm{a}}$ & $\mathbf{D}^{\mathbf{a}}$ & $\mathbf{M}_{n, \text { theo. }}\left[\mathrm{g} \mathrm{mol}^{-1}\right]$ & Yield [mg] & Yield [\%] \\
\hline 1 & PtBAMA & 4000 & 1.46 & 20000 & 190 & 17 \\
\hline 2 & PtBAMA & 3300 & 1.35 & 20000 & 20 & 2 \\
\hline 3 & PtBAMA & 2800 & 1.21 & 20000 & 80 & 7 \\
\hline 4 & $\mathrm{PEO}_{44}-b-\mathrm{P} t \mathrm{BAMA}_{30}$ & 6000 & 1.12 & 22000 & 990 & 41 \\
\hline 5 & $\mathrm{PS}_{30^{-}} b-\mathrm{P} t \mathrm{BAMA}_{40}$ & 7900 & 1.38 & 23400 & 1130 & 81 \\
\hline 6 & $\begin{array}{l}\mathrm{P}_{n} \mathrm{BA}_{25}-b- \\
\mathrm{P}_{t} \mathrm{BAMA}_{50}\end{array}$ & 8100 & 1.18 & 23600 & 1100 & 69 \\
\hline 7 & {$\left[\mathrm{TTP}-\mathrm{P} t \mathrm{BAMA}_{50}\right]_{4}$} & 10900 & 1.15 & 80400 & 216 & 18 \\
\hline
\end{tabular}

a) Determined by SEC [( $\mathrm{CHCl}_{3} / \mathrm{TEA} / i-\mathrm{PrOH}$. (94/2/4): PS calibration]

\section{Equation S1:}

Calculation of the block length ratio for $\mathrm{P} n \mathrm{BA}-b-\mathrm{P} t \mathrm{BAMA}$

$$
n H\left(\mathrm{CH}_{3}, \mathrm{PtBAMA}\right)=\frac{I\left(\mathrm{OCH}_{3}, \mathrm{PtBAMA}\right)}{I\left(\mathrm{CH}_{2}, \mathrm{PnBA}\right)} \cdot \mathrm{N}(\mathrm{H}),\left(\mathrm{OCH}_{2}, \mathrm{PnBA}\right)
$$

